\title{
Des enfants et des livres. Remarques sur des souvenirs d'enfance du monde anglo-saxon
}

\section{Marina Roggero}

Traducteur : Danièle Tosato-Rigo

\section{OpenEdition \\ Journals}

Édition électronique

URL : http://journals.openedition.org/edl/878

DOI : $10.4000 /$ edl. 878

ISSN : 2296-5084

\section{Éditeur}

Université de Lausanne

\section{Édition imprimée}

Date de publication : 15 mai 2016

ISBN : 978-2-940331-48-2

ISSN : 0014-2026

\section{Référence électronique}

Marina Roggero, « Des enfants et des livres. Remarques sur des souvenirs d'enfance du monde anglosaxon », Études de lettres [En ligne], 1-2 | 2016, mis en ligne le 01 mai 2019, consulté le 20 décembre 2020. URL : http://journals.openedition.org/edl/878 ; DOI : https://doi.org/10.4000/edl.878 


\section{DES ENFANTS ET DES LIVRES. REMARQUES SUR DES SOUVENIRS D'ENFANCE DU MONDE ANGLO-SAXON}

Cet article se penche au travers de sources autobiographiques sur la question des lectures d'enfants et d'adolescents dans les communautés puritaines et celles des dissenters (Angleterre et colonies américaines). Comment des lecteurs peu expérimentés se familiarisaient-ils avec les livres à l'époque moderne, et comment s'orientaient-ils dans leur choix de lectures? Peu étudié jusqu'ici, ce cheminement se révélait loin d'être simple, même dans des milieux protestants qui entretenaient un rapport vital, voire quasi obsessif avec les textes religieux et les Saintes Ecritures en particulier. A la différence d'autres sources, littéraires ou normatives, les egodocuments permettent de reconstituer une gamme de comportements individuels et d'appréhender la mémoire de la réalité vécue: celle d'un rapport entre enfants et livres plus diversifié et plus aventureux que ne le font penser les règles du genre et le canon officiel relatif aux premières lectures prescrites au «bon chrétien».

Pour qui s'intéresse aux lectures enfantines, les cercles puritains et ceux des dissenters des deux côtés de l'Atlantique méritent d'être examinés de plus près. Sans doute n'est-ce pas tout à fait sans danger: on court le risque à la fois de généraliser la valeur de témoignages émanant de milieux très spécifiques et d'accentuer le rôle de la Bible en tant que voie d'accès priviégiée à d'autres lectures pour l'ensemble de ses pieux usagers. Mais une fois ce contexte particulier et les limites inhérentes aux sources admis, les mémoires et journaux spirituels émanant de tels groupes présentent par contre le grand avantage d'être accessibles en nombre suffisamment important pour documenter la multiplicité des facettes des parcours individuels. De fait, et c'est bien là que réside tout l'intérêt de la chose, ils permettent de mettre à jour des écarts et des différences 
non négligeables dans des situations où prévalent les mêmes normes, et où s'applique le même canon textuel. Ainsi, la pratique des Saintes Ecritures, fardeau étouffant pour d'aucuns, constituait pour d'autres non seulement une nourriture spirituelle mais un terrain d'apprentissage de l'alphabet et un tremplin pour d'ultérieures lectures.

Prenons pour commencer les exemples de deux vies placées sous le signe des livres, ou plutôt du Livre, telles que les évoquent les écrits d'habitants de Boston de la fin du XVII e et du début du XVIII ${ }^{\mathrm{e}}$ siècle, aisés, cultivés et pieux. Ils éclairent les modalités et les buts de pratiques familiales, tout en en consignant les effets sur les enfants. Dans de tels milieux, on regardait avec horreur les textes de divertissement, qui distrayaient de la vie spirituelle, tels "plays, and songs and novels and romances, and foolish and filthy jests, and poetry prostituted unto execrable ribaldry " ${ }^{1}$. Le projet éducatif s'ancrait inexorablement, dès la plus tendre enfance, dans les textes qui transmettaient la parole divine. Le journal du pasteur Cotton Mather, en particulier les nombreuses pages que ce descendant des Pères Pèlerins consacre à l'éducation de ses enfants, rappelle les règles qui scandaient les journées laborieuses des communautés congrégationnistes: les prières du matin, suivies de la lecture à haute voix et de l'explication d'un passage biblique; la lecture de "some story out of the Bible», donnant lieu à quelque approfondissement moral, qui accompagnait généralement le repas de midi; puis la lecture commentée des Psaumes et la récitation du catéchisme par les garçons, le soir, après les oraisons ${ }^{2}$. Outre ces rendez-vous réguliers, ils avaient d'autres occasions d'étudier et de travailler en commun sur les livres, toujours au niveau spirituel, et toujours dans le cadre d'un travail qui, méthodiquement organisé par le père, privait l'enfant de la moindre initiative. Le géniteur ne se bornait pas à choisir les textes à donner de fois en fois aux enfants, sans jamais les leur laisser, il décidait des chapitres et des pages précises que chacun d'entre eux aurait à transcrire, à commenter ou à lire, à haute voix ou en silence. Ecrivain, polémiste, et protagoniste de l'un des derniers et plus célèbres procès pour sorcellerie intentés dans les colonies américaines (Salem, 1692), Cotton Mather est une personnalité peu commune. Aussi peut-il sembler risqué de renvoyer

I. C. Mather, Betiah, p. 58, cité dans D. D. Hall, Worlds of Wonder, p. 55.

2. C. Mather, Diary; voir J. E. Monaghan, Family Literacy in Early $18^{\text {th }}$ Century Boston, p. 342-370. 
à une telle figure pour saisir ce qui était d'usage courant de son temps. Loin de décrire une vie ordinaire, ses mémoires constituent plutôt le miroir des traits exemplaires du pater familias puritain, un modèle et terme de comparaison pour sa congrégation.

Ceci ne signifie toutefois nullement que les comportements évoqués cidessus étaient atypiques, dans la mesure où on les retrouve chez d'autres membres de ce milieu qui n'ont pas joué le même rôle que C. Mather dans la cité, tout en étant animés de la même foi. Les écrits du for privé du juge et marchand Samuel Sewall en attestent. Confirmant l'extraordinaire importance de l'alphabétisation dans ces familles, ils permettent de faire un pas de plus, en mettant en évidence le problème de la réception, ou pour le moins celui des retombées de cette intransigeante pédagogie du salut. Père et maître, scrutant quotidiennement l'âme de ses enfants dans une recherche des signes de grâce ou de condamnation divine qui tourne à l'obsession, Sewall enregistre en effet soigneusement dans son journal les résultats de l'administration massive de lectures et de pratiques religieuses. En voici un exemple, datant de l'hiver 1690. La triste nouvelle de la mort d'un jeune parent et ami, enlevé par la variole, venait de parvenir à la famille. Sewall en informe immédiatement son fils aîné, profitant de l'occasion pour l'avertir de se préparer très consciemment à la mort, en s'appliquant "really to pray when he said over the Lord's Prayer ${ }^{3}$. Âgé de onze ans, Sam, qui était en train de manger son goûter, semble sur le moment recevoir très froidement la nouvelle et l'avertissement paternel; mais plus tard, lorsqu'il se mit à réciter le Notre Père, le garçon "burst out in a bitter cry and said he was afraid he would die». Son géniteur le soutint avec la plus grande sollicitude: non pour soulager sa peine mais pour prendre soin de son âme immortelle. L'indiscutable affection que Sewall éprouvait pour ses enfants - dont le journal offre de multiples preuves - n'avait que faire d'assurances banales ou d'oubli passager de la douleur; le père voyait dans les moments de crise ou d'angoisse un levier pour le réveil et l'élévation d'ordre spirituel. C'est ce qui explique sa façon d'intervenir auprès de l'enfant: après avoir commencé par inviter son fils à se recueillir en prières, tous deux recourent aux omniprésentes Ecritures pour chercher ensemble dans ces pages l'écho

3. Les extraits du journal de Samuel Sewall sont tirés de J. Monaghan, Learning to read and Write in Colonial America, p. 112 sq. 
de la voix divine "conforting against death, as "O death where is thy sting, etc." ".

Sur la base de telles prémisses, on ne s'étonnera guère de trouver dans la famille de l'irréprochable marchand d'autres manifestations d'inquiétude ou de malaise liées à des lectures. Dans le cas de l'adolescente Betty, ce fut la lecture à voix haute et en famille d'un passage de saint Jean qui provoqua la montée d'une angoisse irrépressible, alimentée par la méditation personnelle d'un sermon de Cotton Mather. Le vers «Ye shall seek me and shall die in your sins - relevait Sewall - ran in her mind and terrified her greatly. [...] A little after dinner, she burst out into an amazing cry, which caus'd all the family to cry too: her mother ask'd the reason; she gave none; at last said she was afraid she would goe to Hell, her sins were not pardon'd». De telles crises de nerfs se répétant, un changement d'air fut jugé bénéfique pour la jeune fille qu’on envoya pour quelque temps chez des parents à Salem. Lors des visites qu'il lui rendait, son père n'en continuait pas moins à lui administrer les habituels et rigoureux remèdes tirés de la Bible: «I set Betty to read Ezek. 37 (The hand of the Lord is upon me [...] and set me down in the midst of the valley which was full of bones) ». Les allusions répétées à la mort, bien qu'atténuées par le message de la résurrection, étaient une source de larmes intarissables, "so that [she] can hardly read"; et aux larmes succédait de temps à autre un douloureux examen de conscience: "She tells me of the various temptations she had - note son père dans son journal - as that she was a reprobat, loved not God's people as she should.»

Pour démêler le sens de cet inquiétant enchevêtrement d'alphabétisation religieuse et d'omniprésence de la mort, il convient de garder en mémoire à quel point les familles puritaines étaient obsédées par l'idée que leurs enfants puissent disparaître en bas âge sans avoir eu le temps de se convertir à Dieu. Ces craintes n'étaient nullement infondées sur le plan démographique, puisque dans la Nouvelle-Angleterre un bon tiers des enfants mourrait avant l'âge de vingt et un an ${ }^{4}$. Les enfants étant considérés comme marqués par le péché originel autant que les adultes, l'alphabétisation précoce et l'étude individuelle des Ecritures constituaient la clé de leur renaissance intérieure, seule à même d'assurer

4. Les pourcentages s'aggravent encore légèrement au siècle suivant, voir D. E. Stannard, The Puritan Way of Death. 
le salut ${ }^{5}$. Mais la chose avait son prix, parfois très élevé. Pour des jeunes filles à l'image de Betty Sewall, qui maîtrisaient bien l'alphabet, le recours intensif aux Ecritures apportait, certes, un réconfort et une nourriture spirituelle, mais il générait simultanément tension intérieure et angoisses. Il ne fait par ailleurs aucun doute, en outre, que dans de tels cas les lectures religieuses formaient un système clos, voire une barrière destinée à isoler les jeunes d'autres univers textuels plus dangereux. Un constat qui invite à interpréter de façon nuancée les hauts taux d'alphabétisation du monde protestant, en évitant de les placer de façon univoque sous le signe de la modernisation, du progrès ou de la promotion de l'individu.

A cet égard, Betty représente probablement un exemple extrême. Son sexe, la figure morale et sociale du père, la richesse même de la maison, tout contribuait à bloquer la situation, réduisant paradoxalement les espaces d'autonomie et de temps libre, de même que la possibilité de rencontres imprévues, puisque chaque moment de la journée demeurait sous le contrôle aussi tendre qu'obsessif de la famille. La lecture n'était toutefois pas aussi verrouillée pour tous, au point que, comme nous l'avons suggéré plus haut, dans d'autres situations les Ecritures pouvaient au contraire représenter un tremplin vers une plus vaste bibliothèque. Il suffit de songer à l'enfance d'un Benjamin Franklin - né en 1706 pour comprendre que la Boston du début du XVIII e siècle offrait aussi des opportunités, du moins pour un garçon éveillé, entreprenant et non dénué de tout. Sa célèbre autobiographie (publiée à titre posthume après $1790^{6}$ ) apporte un utile correctif aux cadres précédemment évoqués, puisque l'atmosphère et l'environnement familial y apparaissent moins étouffants, moins propices à la claustrophobie, et ce nonobstant le fait que le père Franklin ait été un pieux presbytérien, et la vie de son foyer

5. Sur cet univers mental, voir D. Hall, Worlds of Wonder, chap. 5; et J. S. Graham, Puritan Family Life.

6. L'autobiographie de Franklin a circulé sous forme manuscrite parmi ses amis avant sa mort. Des éditions partielles du texte ont été réalisées à partir de 1790. Mais ce n'est qu'en 1868 que ce self-help book extrêmement populaire fut édité en version intégrale. L'édition de tels textes pose une série de problèmes à l'historien (interventions de l'éditeur, scientifique et commercial, etc.). Pour cette contribution nous avons choisi de ne pas distinguer les textes publiés des inédits, dans la mesure où les seconds ont également circulé parmi les amis, collègues ou coreligionnaires de l'auteur, comme le montrent les mémoires de puritains et le confirment les propos de Franklin lui-même. 
pétrie de pratiques religieuses. Le portrait de la famille modeste dessiné par Benjamin Franklin coïncide dans les grandes lignes avec le modèle protestant confirmé aujourd'hui par les historiens de l'éducation: l'apprentissage de la lecture paraît y avoir été naturel, presque imperceptible - «I do not remember when I could not read», note Franklin - et l'expérience scolaire, quoique brève et fragmentaire, semble avoir fait partie de l'horizon commun ${ }^{7}$. Benjamin eut l'occasion de fréquenter d'abord une petite école gérée par une femme (Dame-School), puis, grâce à sa vivacité intellectuelle, une classe de grammaire latine et une autre d'écriture et arithmétique commerciale. Toutefois à l'âge de dix ans il travaillait déjà avec son père, fabricant de chandelles et de savon.

Ses mémoires sont une véritable mine d'informations en ce qui concerne l'accès aux livres. Elles nous apprennent que Franklin fit ses premières découvertes dans la petite bibliothèque familiale, qui consistait, comme il le précise "chiefly of books in polemic Divinity» (incluant Cotton Mather), mais où se trouvaient également quelques œuvres classiques à forte connotation morale, à l'instar des Vies de Plutarque. C'est à partir de tels textes, véritables compagnons d'enfance de Ben, que ce dernier s'aventura plus loin dans les lectures. Il les choisit de façon plus autonome dès qu'il commença à gagner quelque argent. Lorsqu'il se fut pris de passion pour Pilgrim's Progress de John Bunyan - un des rares livres autorisés aux enfants qui fût doté d'une intrigue et d'aventures, certes allégoriques - Franklin se mit en quête de cet auteur ${ }^{8}:$ «My first collection was of John Bunyan' works, in separates little volumes». On observera au passage que le terme de collection ne renvoyait pas à des pratiques d'accumulation ou de thésaurisation. Bien au contraire, Franklin n’hésita pas à revendre son petit stock initial pour investir son bénéfice dans l'achat de nouveaux livres qu'il choisissait principalement dans les articles vendus au rabais par les colporteurs: par exemple les Historical Collections de Richard Burton, pseudonyme de l'éditeur londonien Nathaniel Crouch qui édita à la fin du XVII e siècle des textes à bas prix destinés à un public populaire parmi lesquels une série sur l'histoire

7. B. Franklin, Benjamin Franklin's Autobiography, vol. I, p. 6.

8. John Bunyan (1628-1688), prédicateur baptiste très populaire, auteur prolifique de sermons et traités religieux, publia Pilgrim's Progress en deux parties entre 1678 et 1684. Cette œuvre constitua l'un des plus grand succès de librairie puritains, elle fut largement importée et vendue dans les colonies également. 


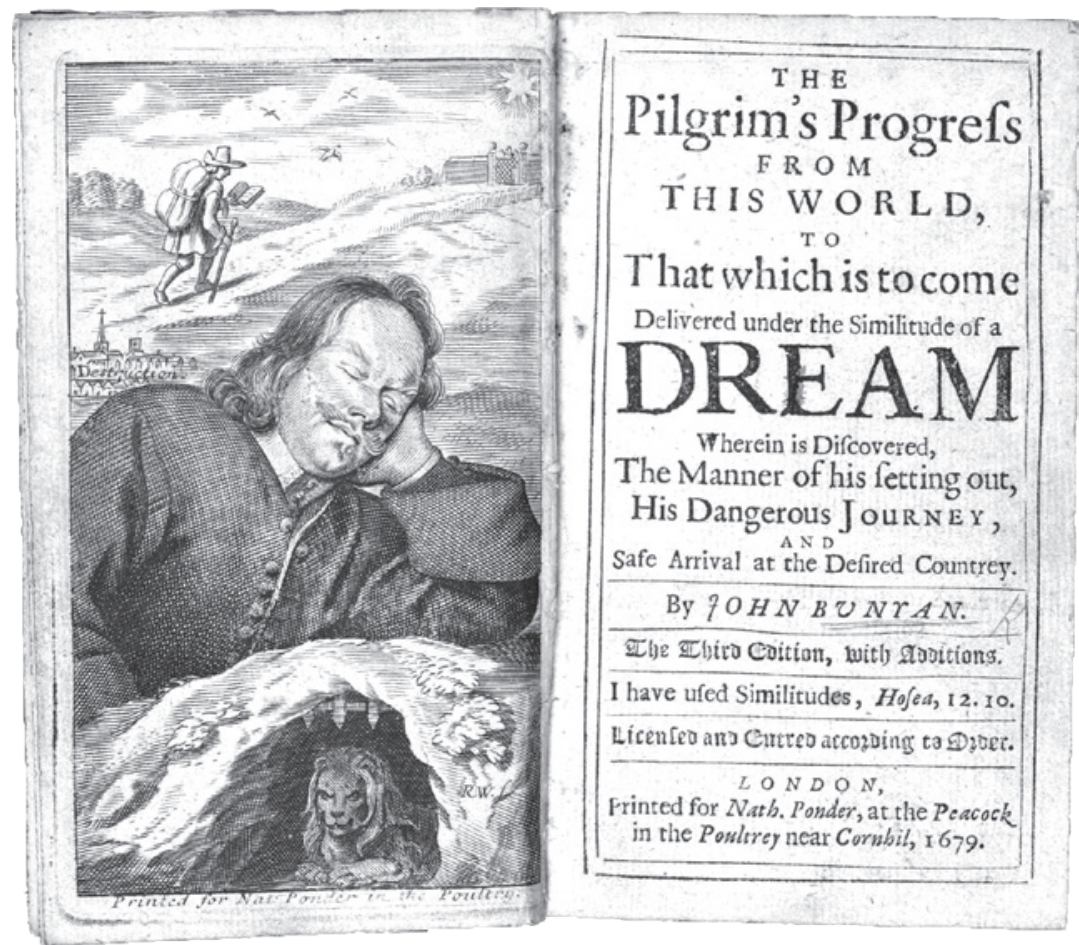

Pages de garde de The Pilgrim's Progress de John Bunyan (1679)

britannique et une autre de récits tirés de la Bible 9 . Il devait en aller tout autrement lorsque Franklin quitta son métier pour la typographie de son demi-frère. Il entra alors de plain-pied dans le monde des livres, non seulement parce qu'il travaillait au milieu d'eux, mais parce qu'il fréquentait des personnes qui partageaient cette passion, des clients jusqu'aux garçons de courses. Le jeune apprenti avait d'innombrables occasions d'en parler, de les échanger ou de les emprunter, sans compter qu'il pouvait se les procurer en cachette en les emportant chez lui le soir pour les rapporter le lendemain, «soon and clean, [...] lest it should be miss'd or wanted» 10 .

Quoiqu'un tel parcours ne soit pas généralisable, comme le montrent les étapes ultérieures de cette existence hors du commun, il fournit

9. R. Mayer, N. Crouch, Bookseller and Historian, p. 391-419.

Io. Benjamin Franklin's Autobiography, p. 10. 
quelques indications qui confirment les contours fragiles et poreux des bibliothèques de ces groupes populaires. On serait tenté de les qualifier de "bibliothèques liquides": leurs propriétaires désargentés se voyaient constamment contraints à remettre en circulation les textes qu'ils s'étaient procurés, ne parvenant à conserver que quelques œuvres auxquelles ils attribuaient une valeur particulière, souvent non commerciale. Plus généralement, et au niveau historiographique, c'est là que semble résider le cœur du problème: dans l'écart existant entre l'importante circulation et la faible conservation des textes. Un écart qui pénalise incontestablement la recherche sur les pratiques de lecture à l'époque moderne. Les échos de contemporains, en laissant entrevoir ces pratiques de diffusion capillaire, attestent du fait que dans l'Angleterre rurale de la fin du XVIII ${ }^{\mathrm{e}}$ siècle notamment, pamphlets et livrets de colportage (chapbooks) étaient disponibles "on every bookstall at fairs and markets", et que cette littérature faisait son entrée dans les villages grâce aux hottes "of every doorcalling hawker". Destinée à la consommation immédiate, cette littérature au rabais, dont les titres étaient «as familiar with everyone a his own name", a laissé peu de traces, contrairement au Book of Hymns, au Book of Common Prayer, à la Bible ou au Nouveau Testament ${ }^{11}$. Même des sources ordinairement aussi précieuses, à certains égards, que les enquêtes officielles, les testaments, les inventaires ou catalogues de libraires ne peuvent guère éclairer cette zone d'ombre. Lectures éphémères, ces pages échangées, empruntées ou transcrites, parfois dérobées ou lues en cachette, n'en valent pas moins la peine d'être prises en compte.

Les mémoires de Franklin fournissent matière à réflexion sur une autre question, non moins intéressante et liée à la thématique examinée jusqu'ici. Elles mettent en évidence une seconde fracture sur laquelle les historiens se sont rarement penchés. Le récit des premiers pas dans l'écriture réalisés par l'auteur du Poor Richard's Almanack ${ }^{12}$ met en effet en lumière le fait qu'être à même d'utiliser une plume pour écrire l'alphabet

II. Voir à titre d'exemple les souvenirs d'enfance du poète paysan John Clare, né en 1793 (mentionnés par D. Vincent, Bread, Knowledge and Freedom, p. 110). Une enquête menée dans le Norfolk trente ans plus tard montre qu'il demeurait rare de trouver dans les maisons d'ouvriers des ouvrages non religieux, alors qu'il n'y manquait jamais un psautier, une bible, le Nouveau Testament et le Prayer-Book.

I2. Publié entre 1732 et 1758 pour instruire et divertir un public populaire, cet almanach constitua l'un des plus grands succès éditoriaux de Franklin. 
ou rédiger des phrases élémentaires était une compétence technique préalable, clairement séparée de la capacité à composer un discours articulé, au même titre que pouvait l'être la capacité à lire des lettres ou des mots de celle de déchiffrer un texte. Le problème ne se posait en règle générale pas pour ceux qui accomplissaient un cursus d'études. Dans les collèges, grammaire, syntaxe et rhétorique faisaient l'objet d'un apprentissage fondé sur les modèles antiques - et en particulier sur Cicéron, analysé, médité et reproduit de manière presque obsessive - qu'on appliquait par imitation au langage vernaculaire, en recourant, le cas échéant, au canon élevé de la littérature nationale, selon le lieu, on fit une place de plus en plus importante. Mais tous ceux qui s'arrêtaient au seuil de tels établissements éprouvaient les plus grandes difficultés à maîtriser l'usage de la langue écrite. Et ce, quand bien même, comme Ben, ils étaient assoiffés de savoir et nullement illettrés. Au fil d'un parcours tortueux, qui n'avait cependant rien d'inhabituel pour les autodidactes de l'époque moderne, c'est la composition poétique qui lui servit de banc d'essai ${ }^{13}$. Tout bien pesé, elle était d'accès plus facile. Et ce, parce qu'elle s'avérait liée à une mémoire communautaire et collective centrée sur un stock de rimes et de chansons susceptibles de captiver indépendamment de la familiarité de ses usagers avec la lecture. Outre de prières, les proverbes se nourrissaient d'avertissements moraux et d'avis pratiques de toute sorte, pour la plupart rythmés ou allitérés afin d'être plus facilement appris par cœur. Comme l'expliquait un témoin anglais du début du XVIII siècle, les vers «stick faster in the memory than prose», et "the clinking of syllables» touchaient et fascinaient tout particulièrement «the ordinary people» ${ }^{14}$. Vite apprises, vite répétées, les rimes - souvent mises en musique - ne servaient pas qu'à propager l'information, la satire ou des avis divers; elles constituaient une forme populaire de divertissement rythmant les moments du travail et de loisir. L'important potentiel du medium poétique était au reste bien connu des contemporains. D'innombrables auteurs de satires, libelles ou messages politiques, plus

I3. Sur les working class poets, voir E. P. Thompson, Customs in common, et W. J. Christmas, The Lab'ring Muses.

I4. Cité dans A. Fox, Oral and Literate Culture in England, p. 24. Voir également T. Watt, Cheap Print and Popular Piety. 
intéressés à la diffusion rapide et efficace de leur propos qu'à sa qualité littéraire, y recouraient ${ }^{15}$.

"I took a fancy to poetry and made some little pieces», rappelait quant à lui Franklin, qui avait aussi composé et vendu dans la boutique de son frère une ballade inspirée de l'actualité. Mais le fait que la poésie ne permette guère de survivre - bien au contraire, comme l'en avertissait son père, "verse-makers were generally beggars" - convainquit le jeune homme, qui ne manquait pas de sens pratique, de délaisser les rimes pour la prose. C'est alors que devaient émerger les problèmes irrésolus d'une formation qui sur le papier semblait plutôt solide, et qui était incontestablement meilleure que celle dont pouvaient se targuer la plupart des jeunes de son âge. A savoir que ni l'année passée dans une école «for writing and arithmetic», ni son apprentissage de typographe, au cours duquel il avait pourtant parfait son épellation et sa ponctuation, ne lui avaient donné une réelle maîtrise de l'art de l'écrire. Dès qu'il s'agissait de rédiger un texte complexe ou de composer un discours argumenté, ses lacunes sautaient aux yeux; loin de se limiter à l'élégance formelle, elles avaient trait au manque de méthode et de clarté dans l'exposition. Un enseignement au niveau élémentaire ou une formation en autodidacte ne suffisaient en règle générale qu'à l'acquisition d'un savoir instrumental, allant de la maîtrise des écritures courantes à celle des calculs indispensables à la vie quotidienne et professionnelle; au-delà de cette alphabétisation basique, en revanche, le passage devenait étroit, et la sélection d'autant plus dure que les étapes suivantes marquaient un tournant, un bond en avant dans le parcours de formation. Entreprendre des études coûtait cher et, s'agissant d'une voie d'accès à une potentielle ascension sociale, demeurait réservé à une minorité.

Vers quel modèle, et surtout vers quelles lectures pouvait dès lors se tourner, hors cadre scolaire, une personne aussi pleine d'initiative que celle qui nous occupe, fermement décidée à enrichir son vocabulaire et à se doter d'un style efficace? Si en ce début de XVIII ${ }^{e}$ siècle, syllabaires et livres de lecture pour débutants ne manquaient pas, il s'avérait plus difficile de trouver un ouvrage distillant ce que Franklin entendait apprendre ${ }^{16}$. Aussi n'est-ce pas un hasard si son livre d'exercices fut un journal, plus précisément l'une des premières revues de divertissement

15. A. Fox, Ballads, Libels and Popular Ridicule in Jacobean England.

I6. J. Monaghan, Learning to Read and Write. 
et de critique des mœurs apparue sur le marché: "About this time I met with an odd volume of the Spectator", devait-il déclarer dans ses mémoires; "I bought it, read it over and over, and was much delighted with it». Ce choix du "Spectator» est singulier mais très intéressant, d'abord parce qu'il n'a pas été programmé mais découle d'une rencontre fortuite - la découverte d'une série dépareillée du périodique entretemps disparu ${ }^{17}-$, mettant ainsi en évidence combien le hasard et la modicité du prix influençaient l'acheteur. Ceci dit, et malgré ces facteurs déterminants, il s'agissait d'une décision parfaitement sensée. Le langage à la fois simple, subtil et efficace du périodique d'Addison, the familiar way of writing qui en avait assuré le succès auprès d'un public réunissant diverses couches sociales en faisait un excellent terrain d'exercice pour lecteurs et écrivains débutants: "I thought the writing excellent, and wished if possible to imitate it", relève Franklin. Autre élément intéressant à relever dans ce passage autobiographique: il consigne minutieusement la série d'opérations effectuées sur la base du texte-modèle. En dehors des heures de travail, bien évidemment: «My time for these exercises and for reading was at night after work, or before work began in the morning, or on Sundays when I contrived to be in the Printing House alone» ${ }^{18}$. Durant ces heures soustraites au repos ou au divertissement, Ben commençait par rédiger avec la plus grande précision possible des fiches reprenant certaines parties d'articles, dont il s'appropriait la forme et le contenu; puis, après avoir laissé reposer le tout pendant quelques jours, il s'essayait à la recomposition de l'original à partir de ses notes, en relevant après coup erreurs et imprécisions. Pour augmenter la difficulté de l'exercice, il avait aussi pour habitude de mélanger ses notes avant de les réorganiser dans un nouvel ordre logique; enfin, pour enrichir son vocabulaire et affiner son style, il s'imposait de transformer des bouts du texte de référence en vers, qu'il remettait en prose après un certain laps de temps.

Cet obsessif travail de déconstruction, de reconstruction et d'ajustement démontre en tout premier lieu la trempe du protagoniste: sa lutte

17. Publié entre 1711 et 1714 , le Spectator connut un succès prodigieux non seulement en Angleterre mais sur le continent et en Amérique. Lorsqu'il cessa de paraître, les rééditions de ses numéros en volumes continuèrent à circuler sous la forme d'innombrables copies.

I8. Benjamin Franklin's Autobiography, p. 11 sq. 
obstinée avec les mots l'érige en personnification exemplaire de la vertu de la jeune Amérique, entreprenante, combative et ingénieuse. De façon plus générale, l'histoire de son succès s'apparente toutefois à celle d'autres vies plus obscures. Plusieurs autobiographies émanant des couches populaires (rédigées en nombre croissant dès la fin du XVIII siècle) rappellent les efforts et les sacrifices demandés aux classes laborieuses pour forcer ce passage. Elles témoignent, au-delà de toute amplification littéraire ${ }^{19}$, de l'effort consenti pour apprendre les règles du code si bien protégé de l'écriture formalisée.

Certes, pour en revenir à notre propos, les obstacles à vaincre étaient moindres, et la récompense émotionnelle ou matérielle plus immédiate pour qui se limitait au domaine de la lecture ou d'une écriture cursive fonctionnelle. Sur ce terrain, précisément, il peut s'avérer utile de confronter les mémoires de Franklin à d'autres récits de vies ordinaires, pour la plupart hors des milieux des puritains et des dissenters. Un coup d'œil aux mémoires rédigés au XVIII ${ }^{\mathrm{e}}$ siècle par des scripteurs sans cursus scolaire montre que dans le Royaume-Uni aussi, les principaux ouvrages donnés à des enfants, et les premiers mis entre leurs mains étaient en règle générale les mêmes que ceux que Ben avait trouvés dans la maison paternelle: la Bible, les Psaumes, les œuvres de Bunyan, auxquels venaient parfois s'ajouter des pamphlets de propagande ou de polémique religieuses ${ }^{20}$. Dans le lot d'ouvrages mentionnés par Franklin dans les premières pages de son autobiographie, une absence mérite d'être relevée, celle du Paradise Lost de John Milton ${ }^{21}$, texte clé dans leur monde, autant sous l'angle religieux que littéraire, signalé en revanche avec une fréquence significative même dans les maisons les plus modestes du vieux continent.

Le consensus règne par contre en ce qui concerne le Pilgrim's Progress, très apprécié par le jeune Franklin, comme dans la mère patrie où il se diffusa par capillarité. La fascination exercée sur les jeunes lecteurs par cette œuvre, qui traversa les siècles, ressort clairement des souvenirs du londonien William Hone, né en 1780. Un cas particulier susceptible de

19. Voir à ce sujet les recherches de D. Vincent, en particulier Bread, Knowledge and Freedom, ainsi que "Reading Made Strange», p. 181-197.

20. M. Spufford, «First Steps in Literacy».

2I. La première édition de l'épopée en vers libres de John Milton, inspirée de l'histoire d'Adam et Eve, date de 1667. 
donner quelque relief au vernis lisse des généralisations. Il permet en particulier de mettre en relation l'accueil enthousiaste fait au Pilgrim's Progress par Hone avec l'horizon d'attente du jeune homme, et avec les alternatives concrètement à sa portée en matière de lectures. Le point de départ réside dans la très modeste bibliothèque familiale. Ce n'est qu'à l'intérieur de ce contexte, et au regard des éléments recueillis jusqu'ici, que l'histoire bien-aimée du bon pèlerin en voyage vers la cité céleste prend tout son sens.

A en croire Hone, en dehors des Ecritures saintes, il y avait très peu de textes chez lui, et aucun qui soit particulièrement adapté ou simplement un tant soit peu attractif pour un jeune garçon. L'auteur évoque une copie mutilée du Paradise Lost de Milton, un vieux volume de maréchalerie hérité de ses grands-parents, un livre, en plus mauvais état encore, de recettes de cuisine utilisé par sa mère, auxquels s'ajoutaient quelques pamphlets théologiques dus au pasteur de la congrégation à laquelle la famille appartenait: un type d'imprimé éphémère du point de vue bibliographique - il est rarement recensé par titre ou par auteur - malgré sa place tout sauf marginale dans les esprits et les coeurs, et sur les étagères des groupes dissenters (foyer Franklin compris). Pour comprendre l'attrait exercé sur le jeune Hone par l'œuvre de Bunyan, il suffit de songer aux limites quantitatives et qualitatives de la collection familiale, comme au fait que l'acquisition de l'oeuvre est mentionnée par l'auteur comme un événement ayant marqué son enfance:

The addition of such a book as the Pilgrim's Progress to such a collection as ours was to me an event. [...] All the cuts were rude, yet they all pleased me; but the pleasure I derived by the work itself is indescribable. I read in it continually, and read it through repeatedly ${ }^{22}$.

Qu'un enfant volontaire et affamé d'histoires, mais dépourvu de tout bagage culturel solide, ait pu éprouver un tel enthousiasme pour les aventures du héros chrétien et pour sa quête de salut conforte la thèse selon laquelle l'histoire du Pilgrim's Progres parvint à toucher un large public qui comprenait «even the careless or uninstructed readers» ${ }^{23}$.

22. Les extraits des Memoirs of Childhood de Hone sont tirés de L. D. Queeny, Fiction and the Reading Public, p. 110.

23. E. P. Thompson, The Making of the English Working Class, p. 34. Selon une telle interprétation, le livre de Bunyan, instrument d'évangélisation des groupes populaires 
Comparable au grand poème épique de Milton, mais plus largement accessible, la prose sans fioritures de Bunyan puisait dans une langue et dans des images allégoriques doublement captivantes: aisément identifiables et rassurantes, car tirées directement de la Bible, elles avaient aussi le pouvoir de donner un sens aux événements, dans la mesure où on les interprétait à la lumière de la vision religieuse empreinte du millénarisme qui était encore très répandue dans les milieux populaires ${ }^{24}$.

La présence très diffuse de lectures religieuses, telles celles que nous venons de mentionner, et dont il paraît difficile de surestimer l'influence, ne doit toutefois pas faire oublier les possibilités d'accès à des textes plus profanes, qui pouvaient se révéler complémentaires à la lecture d'édification ou au contraire entrer en opposition avec elles. Dans la seconde moitié du XVIII ${ }^{\mathrm{e}}$ siècle, cette tendance se propagea peu à peu, même au sein du public peu cultivé, où elle finit par être assumée de façon plus ouverte et désinvolte. Penchons-nous par exemple sur le récit de Stephen Allen, pauvre apprenti qui passa sa jeunesse dans la New York de la révolution. Ayant quitté l'école pour travailler alors qu'il avait à peine douze ans, il ne bénéficiait que d'une instruction limitée: il savait lire et écrire, connaissait les règles de base en arithmétique, mais "possessed no knowledge of grammar, and was wretchedly difficient in my spelling ${ }^{25}$. Or, cet enfant d'une veuve ruinée - et autre self made boy capable de triompher de toutes les difficultés - aimait passionnément les livres. Non seulement, il collectionnait les textes scolaires, y compris le Nouveau Testament ou "some of Dilworth's texts" ${ }^{26}$. Mais il chassait sans relâche l'imprimé d'occasion ou au rabais, ne pouvant s'en offrir d'autres. C'est ainsi, soit en tentant de mettre à profit les opportunités qu'offrait un marché éditorial en pleine croissance ${ }^{27}$, qu'Allen parvint

dans un premier temps, serait ensuite devenu, à côté du livre de Thomas Paine Rights of Men (1791), un des textes fondateurs du mouvement de la working-class anglaise après 1790 , renforçant les liens qui rattachaient les luttes religieuses du XVII ${ }^{\mathrm{e}}$ siècle à celles de nature économique et politique du XVIII .

24. Ch. Hill, A Turbulent, Seditious and Factious People.

25. Sur Allen, qui devint maire de New York de 1821 à 1824, voir C. F. Kaestle, Pillars of the Republic, p. 31.

26. Il s'agissait de populaires livres d'orthographe et sans doute de deux œuvres de Thomas Dillworth qui eurent un grand succès en Angleterre et dans les colonies: The English Instructor (1728) et A New Guide to the English Tongue (1740).

27. Voir H. Amory, D. D. Hall, A History of the Book in America. 
progressivement à passer de la littérature didactique et religieuse aux œuvres de divertissement, puis, glissant sur des pentes de plus en plus dangereuses, aux "books of a pernicious tendency", sur lesquels il ne donne malheureusement pas davantage de précisions. Quoi qu'il en soit, l'essentiel de ses lectures demeura longtemps ancré dans les «old plays, novels, songs, poetry, history", un matériau qui dans le monde anglosaxon représentait une sorte d'alternative, voire de compensation aux lectures canoniques. Enseignants et moralistes - parmi lesquels Thomas Dilworth mentionné ci-dessus - avaient prêché sans relâche, et continuaient leurs attaques contre cette littérature "de bas étage», ordonnant d'en tenir éloignés en particulier les jeunes et les lecteurs inexpérimentés. Pourtant, indépendamment des condamnations, la diffusion capillaire dont faisaient l'objet les «idle pamphlets, lewd plays, filthy songs, and unseemly jests " ${ }^{28}$ rendait difficile la prohibition de ces "merry books» qui dans leur ensemble constituaient l'autre irrépressible visage de la littérature, ancré dans le plaisir et le divertissement.

Tendance depuis toujours réprouvée des deux côtés de l'Atlantique, le mélange des lectures avait beau être vigoureusement condamné, il n'en exerçait pas moins une irrésistible attraction sur le public. L'auteur du Pilgrim's Progress en personne, John Bunyan, a avoué avoir dans sa jeunesse préféré les ballades, vieilles fables et romans chevaleresques ${ }^{29}$ aux Ecritures saintes: une preuve, si besoin était, de l'irrésistible attrait qu'exerçaient ces lectures tout public. En Angleterre, en particulier, des maisons d'édition spécialisées contribuèrent à leur diffusion et à leur succès au cours du XVIII e siècle. L'offre s'étendait par capillarité aux zones rurales grâce à l'action des colporteurs et des maîtres ambulants "with bundles of trashy pamphlets and orations on his back, scattering [...] miserable wares through all the cottages and workshops and kitchens in the country» ${ }^{30}$. Riches en témoignages sur les chemins tortueux

28. Citation de Thomas Dilworth, dans J. Monaghan, Learning to Read and Write, p. 227.

29. "Give me a ballad, a new-book, George on Horseback or Brevis of Southampton, give me some book that touches curious arts, that tell old fables; but for the Holy Scriptures I cared nor". Citation de Bunyan dans M. Spufford, Small Books and Pleasant Histories, p. 7.

30. G. M. Duval, Littérature de colportage. La citation est tirée de C. F. Kaestle, "Between the Scylla of Brutal Ignorance and the Charybdis of a Literary Education", p. 183. 
empruntés par les livres, les documents autobiographiques font eux aussi écho à ce phénomène. Ainsi que nous l'avons relevé plus haut, au fil du temps, et à mesure qu'ils se dégageaient des entraves du modèle édifiant de la conversion spirituelle, les récits de vie ménagèrent une place plus importante à la découverte de la lecture, relatée de façon plus articulée et plus complexe. C'est, au miroir de la vie privée, une transformation plus générale qu'ils esquissent. Elle tient moins à la disparition des anathèmes contre la lecture profane - répétés à l'envi et avec une vigueur croissante par les moralistes intransigeants, et périodiquement attisés par les réveils religieux ${ }^{31}$ - qu'à la diminution de leur pourvoir d'action sur une société en mutation, où s'affirme un nouveau climat: un climat favorable à une recomposition partielle de la dichotomie entre livres de piété et de divertissement (godly et merry books) propice à la multiplication d'usages diversifiés, même dans les milieux populaires. C'est précisément au cours de ces décennies que s'esquissa une sorte de sécularisation de la tradition des autobiographies spirituelles, à laquelle nous avons déjà fait allusion, tradition née au cours du $\mathrm{XVI}^{\mathrm{e}}$ siècle parmi les groupes puritains qui dut son extension, un siècle plus tard, au succès des mouvements méthodistes. Peu à peu s'attelèrent à ce genre littéraire des scripteurs de divers milieux, animés de motivations très différentes, mais liés par une vision commune plus optimiste de la nature humaine, de même que par un intérêt manifeste pour les aspects séculiers de l'existence terrestre. Le courant des working class autobiographies révèlera par ailleurs une autre tradition durable, celle du récit oral, autrefois alimenté à des titres divers par des écritures éphémères (ballades et feuilles volantes), désormais récupéré dans des structures formalisées d'enregistrement de l'expérience individuelle ${ }^{32}$.

Tant la superposition de textes de genre et de qualité variables que la persistance de l'imbrication entre culture orale et écrite apparaissent de façon visible dans le cas de James Raine, un érudit local né à la fin du XVIII ${ }^{\mathrm{e}}$ siècle dans le Yorkshire. L’Angleterre de George III de Hannovre, rappelons-le, était caractérisée par des taux élevés d’alphabétisation et par une grande vitalité économique et culturelle. Il suffit, pour s'en

3I. L'impact du réveil méthodiste sur le monde du livre est attesté au cours du siècle par la présence en forte augmentation des sermons et des Magazines de John Wesley dans les bibliothèques populaires. L. D. Queeny, Fiction and the Reading, p. 97 sqq.

32. Sur ces aspects, voir D. Vincent, Bread, Knowledge and Freedom, chap. 2. 
convaincre, de songer aux centaines de milliers de copies des Rights of man distribuées par Thomas Paine dans les années 1790 auquel l'establishment politico-religieux répondit en jetant gratuitement ou à bas prix sur le marché quantité de contes et de ballades moralisatrices ${ }^{33}$. Dans ses mémoires, James Raine se décrit comme un garçon pauvre dont la grand-mère vivait à la maison de charité. Pourtant son père était un petit artisan rural, qui exerçait le métier de forgeron, quant à sa mère, simple couturière, non seulement elle savait lire, mais écrivait assez bien pour être considérée "as the great scholar of the village, writing letters for fathers and mathers to their sons and theirs relations» ${ }^{34}$. En tous les cas, sa famille réussit à lui assurer une instruction de base, quoique bricolée comme c'était l'usage dans les classes populaires. De trois à cinq ans, l'enfant fut placé dans différentes Dames-schools tenues par des domestiques ou des femmes de journaliers, simultanément gardiennes et éducatrices; le maître qui leur succéda ne pouvait guère se targuer de meilleures références: cet ancien manœuvre réunissait les élèves dans une cabane couverte de paille. Et pourtant: de ces écoles improbables Raine sortit capable d'écrire et de lire "almost anything that fell my way» 35 .

Etudes mises à part, il est intéressant de constater comment à un noyau de textes sacrés prônés sans relâche par ses éducateurs et sur la base duquel le garçon apprit l'alphabet, vinrent peu à peu s'ajouter diverses autres lectures. Si la grand-mère maternelle de Raine, pauvre et aveugle, n'avait pu lui apprendre par cœur que les hymnes d'Isaac Watts, son aïeule paternelle, par contre, disposait d'une petite bibliothèque à laquelle le jeune homme eut librement accès. Elle contenait une ancienne vie du Christ, imprimée au début du XVIII siècle, "ornamented at the head of each chapter with a rude woodcut». Mais le joyau de la collection, du moins aux yeux de l'enfant qui en feuilletait inlassablement les pages "with infinite delight", était un exemplaire des Fables d'Esope, «tattered and torn and imperfect, equally ornamented with

33. En ce qui concerne le Cheap Repository of Moral and Religious Tracts evangélique, actif entre 1795 et 1798, S. Pedersen parle de non moins de deux millions d'exemplaires («Hannah More Meets Simple Simon», p. 84-113).

34. Pour l'autobiographie de Raine, voir M. Spufford, "Women teaching Reading to Poor Children", p. 47-62.

35. Ce n'est qu'en commençant une grammar school, à l'âge de quinze ans, que Raine se mit à l'apprentissage du latin. 
woodcuts» ${ }^{36}$. Outre des ouvrages, en nombre restreint, l'aïeule possédait une immense collection ("an immense bundle») d'imprimés éphémères: des brochures, petits contes, feuilles volantes ou histoires à un sou (penny histories) qui reprenaient et vulgarisaient des genres divers, des contes de fées, de sorcières ou de géants, aux exploits de héros du temps passé. Le prix très modeste de cette forme éditoriale à l'aspect volontairement rudimentaire en explique la pénétration dans les foyers les plus modestes. Elle suscitait souvent la curiosité et l'intérêt de lecteurs débutants, comme le rappelle précisément le cas de Raine. Ayant pratiqué ce genre de lectures, le jeune homme connut bientôt sur le bout des doigts aussi bien les ballades de Robin Hood qu'un roman chevaleresque censé répondre à un genre plus noble tel The seven Champions of Christendom ${ }^{37}$ (dont il ne précise malheureusement pas la version utilisée). Il convient de rappeler à ce sujet que témoignages et souvenirs autobiographiques font émerger par fragments un phénomène peu visible à distance, mais dont les historiens du livre signalent la portée : à savoir que contrairement aux vies du Christ ou aux fables d'Esope - ou autres ouvrages de référence aux contenus divers -, qui avaient des bonnes chances d'être transmis à la postérité et répertoriés par les chercheurs, les bundles of merry histories transversalement diffusés auprès de lettrés comme de semi-alphabètes tendaient en revanche à laisser peu de traces, aussi bien dans les bibliothèques que dans la mémoire collective, parce qu'ils sombraient dans un silencieux naufrage bibliographique. Une perte matérielle qui n’efface pas le fait que pour nombre d'enfants de divers milieux, ces textes écoutés ou déchiffrés individuellement représentaient l'un des premiers liens avec le monde de l'écrit, et la base minimale à partir de laquelle jailliraient peutêtre d'autres curiosités ou d'autres lectures.

Les premières expériences de Raine peuvent être comparées avec celles d'un autre jeune usager d'une bibliothèque assemblée de façon fortuite dans les mêmes années. Il s'agit de Thomas Carter, né en 1792, issu lui aussi d'un milieu modeste, et futur tailleur de profession. Point n'est besoin de dire que l'accès aux livres eut lieu pour Carter à travers la voie habituelle, celle des textes religieux possédés par la famille: «My parent's

36. M. Spufford, Small Books and Pleasant Histories. La diffusion des fables d'Esope était liée à son usage scolaire, tout comme au manque de littérature enfantine qui en faisait un livre qui plaisait aux enfants.

37. M. Spufford, Women Teaching, p. 51. 
stock consisted of two Bibles, a Common Prayer Book, a Universal Spelling Book, Watt's Divine and Moral Songs, with some tattered and old volumes of sermons and other theological disquisitions ${ }^{38}$. Sur ces pages, le jeune homme apprit l'alphabet et commença à déchiffrer les mots: rien que de très normal pour son temps et dans son milieu. Moins prévisible, et peut-être plus intéressant est le récit de sa découverte de la littérature profane. C'est une vendeuse de friandises, qui débitait outre des «cakes, sweetmeats and fruit» aussi des «little books» qui devaient lui offrir la possibilité d'avoir entre les mains une lecture divertissante. Le garçon n'avait pas le sou, mais il parvint à gagner la sympathie de la vieille marchande, ce qui lui permit de lire sans bourse délier «all the little stories which she kept on sale». Une deuxième chance lui fut offerte par des camarades de classe. Sa mère ayant ouvert une petite Dameschool pour vivre, Thomas, en la fréquentant eut la possibilité de découvrir et de feuilleter "several small books belonging to the children, which otherwise would not have come in my way». Entré ensuite dans une école gérée par une congrégation de dissenters, Carter trouve une nouvelle fois comment accéder aux bibliothèques des autres, en obtenant le droit de puiser dans celle de son maitre. "The books were chiefly old and odd volumes of the "Arminian" and the "Gentleman's" Magazines", note-t-il dans ses mémoires. Il ne s'agissait donc pas de livres, à proprement parler, mais de numéros de vieilles revues encore en circulation que l'on conservait au même titre que des ouvres d'auteurs. Quoique l'Arminian Magazine était de tendance moralisatrice et didactique, et le Gentleman's Magazine littéraire et mondain, tous deux réunissaient vers et sermons aux anecdotes et extraits divers ${ }^{39}$. Carter souligne que malgré leur «little intrinsic value, [they] were to me a treasure». Il ajoute que les deux revues firent de lui un "undiscriminating reader », prêt à dévorer la moindre page qui tombait sous ses yeux, aussi indigeste qu'elle puisse être. L'étape suivante, et la dernière à nous intéresser ici, s'effectue dans la boutique où Carter entra en service. Evidemment, notre apprenti sut là encore convaincre son jeune maître au cœur généreux de l'autoriser

38. Les citations des Memoirs of a Working Man de Thomas Carter sont tirées de D. Vincent, Bread, Knowledge and Freedom, p. 116-118.

39. L'Arminian Magazine, lancé en 1778 par John Wesley pour promouvoir la diffusion du méthodisme s'ouvrit par la suite aux sciences et à la littérature. Le Gentleman's Magazine, autre périodique littéraire à grand succès, fut fondé en 1731 par le journaliste Edward Cave. 
à utiliser sa petite bibliothèque. Elle réunissait ouvrages instructifs (de diction, d'histoire et géographie), volumes de poésie et quelques romans, non des plus récents, tel The Vicar of Wakefield paru en $1766^{40}$. Thomas Carter fut peut-être un garçon particulièrement curieux et chanceux dans ses relations avec autrui, de même que les personnes qui l'entourèrent se montrèrent peut-être particulièrement généreuses envers lui; il n'en reste pas moins que son parcours d'apprenti lecteur évoque l'existence de vastes réseaux et de pratiques communes d'échange, en un mot l'existence d'une sociabilité de l'écrit développée même dans des milieux non élitaires et qui revêtait des dimensions et une densité difficiles à imaginer de nos jours.

De telles opportunités ne doivent pas être perdues de vue, dans la mesure où elles représentaient un moyen efficace de pallier à l'absence de matériau écrit de même qu'à son coût prohibitif. Il s'agissait toutefois de bricolage, de solutions de compromis, peut-être fonctionnelles sur le plan pratique, mais insuffisantes à abattre les obstacles idéologiques et politiques que rencontrait tout ce qui était perçu comme une avancée déplorable et dangereuse des semi-lettrés. Stopper les outsiders dépourvus de légitimité sociale qui ne se contentaient pas des premiers rudiments du savoir et qui prétendaient accéder à une culture livresque jugée hors de leur portée devait pour longtemps représenter un objectif propre à cimenter les hiérarchies laïques et spirituelles. Avant la fin de l'Ancien régime, mais après également, jeunes et adultes des classes populaires ne pouvaient que difficilement concilier les obligations professionnelles avec le goût de la lecture désintéressée, sans compter l'énergie qu'ils devaient mettre à vaincre la suspicion dont ils étaient l'objet, de la part de leurs supérieurs, ou même de leurs parents et amis. Dans les cercles d'ouvriers et d'artisans anglais du XIX ${ }^{\mathrm{e}}$ siècle, milieux dans lesquels l'alphabétisation était largement répandue et bien acceptée, l'intérêt pour les œuvres littéraires, ou non directement utiles, était souvent considéré comme inapproprié, abstraction faite de la valeur intrinsèque du texte lui-même. Un témoin s'en souvient, en évoquant son dur combat pour arriver aux livres dans une famille en proie aux difficultés matérielles: «My

40. "My young master [...] gave me free access to his little library, in which were Enfield's "Speaker”, Goldsmith's "Geography”, an abridged "History of Rome”, a "History of England", Thomson's "Seasons", "The Citizen of the World”, “The Vicar of Wakefield", and some other books the titles of which I do not remember." 
grandfather, catching sight of me reading a copy of [...] Shakespeare, sternly reproachful, exclaimed: "Ah Tom, that'll never bring you bread and cheese" ", écrit Thomas Okey ${ }^{41}$.

Au-delà de cas singuliers, une leçon que l'on peut tirer des sources autobiographiques est qu'il convient d'être prudent, et d'utiliser des grilles d'interprétation flexibles dans l'étude des lectures et des lecteurs, tout particulièrement de milieux populaires. Malgré son attrait, une enquête fondée sur les critères d'ouverture à la nouveauté et de réception immédiate d'œuvres à succès risque d'occulter l'importance d'un noyau dur de textes détachés de toute recherche d'information et de nouveautés, dont on sait néanmoins qu'ils furent consommés avec avidité. Hommes et femmes en contact occasionnel avec les livres, par manque d'argent ou en raison de leur style de vie, se voyaient dans l'obligation de thésauriser chaque page qui leur tombait sous la main, maintenant ainsi un rapport souvent vital avec des œuvres - ayant ou non trait à la religion - passées de mode et disparues du marché. A côté des dates de parution ou du nombre d'exemplaires mis sur le marché, d'autres éléments doivent donc être pris en compte, liés à la durée de vie des textes et aux formes plurielles de leur utilisation et réutilisation.

Par ailleurs, comme on l'a déjà rappelé, les principes de cohérence et d'organicité ne s'appliquent que partiellement à un tel type de collections de textes, qui étaient davantage le fruit du hasard ou d'opportunités diverses que le résultat de décisions prises au préalable. Ce caractère imprévisible et les méandres multiples empruntés par les lectures diminuaient dans une certaine mesure l'efficacité du contrôle exercé par les censeurs, officiels ou domestiques, qui ne trouvaient pas toujours le moyen de bloquer les parcours sortant de la norme ou d'empêcher les rencontres accidentelles. Ceci dit, ces effets collatéraux ne changeaient rien au problème de fond: à savoir que les personnes démunies et ayant un nombre limité de relations avaient bien peu de possibilités de choisir ce qu'elles lisaient, dans le domaine religieux et plus encore pour ce qui était des textes profanes. Les conséquences que cela pouvait avoir variaient bien entendu selon les périodes, les lieux et la personnalité des individus eux-mêmes. Si l'optimisme conquérant avec lequel un jeune

4I. La citation des mémoires de Thomas Okey, né en 1852 dans une famille de condition très modeste, et devenu professeur d'italien à l'université de Cambridge, est tirée de D. Vincent, Literacy and Popular Culture in England, p. 209. 
homme comme Benjamin Franklin se frayait un chemin dans la vie ne fut - en apparence pour le moins - nullement entamé par son aventureuse et anarchique chasse aux livres, d'autres, moins chanceux ou moins sûrs d'eux, ressentaient cette incohérence et les efforts entrepris comme beaucoup plus pénibles. Le tisserand et poète par passion William Thom, né en Ecosse à la fin du XVIII ${ }^{\mathrm{e}}$ siècle, orphelin de père, qui n'avait fait qu'un bref passage dans une Dame-School avant de devoir travailler dès l'âge de dix ans, écrivait non sans amertume: «My books - I have few of my own - pick up a loan where it can be had». Et de conclure: «so of course my reading is without choice or system ${ }^{42}$.

Marina Roggero

Università degli Studi di Torino Traduit de l'italien par Danièle Tosato-Rigo et Damiano Bardelli

42. Cité par D. Vincent, Bread, Knowledge and Freedom, p. 118. 


\section{BIBLIOGRAPHIE}

Amory, Hugh, Hall, David D. (eds), A history of the book in America, Worcester/Massachusetts, American Antiquarian Society, 2010.

Burnett, John, Destiny Obscure. Autobiographies of Childhood, Education and Family from the 1820's to the 1920's, Harmondsworth, Penguin Books, 1984.

Cavallo, Guglielmo, Chartier, Roger (dir.), Storia della lettura, Roma, Laterza, 1995.

Chartier, Roger (dir.), Histoires de la lecture. Un bilan des recherches: actes $d u$ colloque des 29 et 30 janvier 1993, Paris, IMEC/Editions de la Maison des Sciences de l'homme, 1995.

Christmas, William J., The Lab'ring Muses: Work, Writing, and the Social Order in English Plebeian Poetry, 1730-1830, Newark/ London, University of Delaware Press/Associated University Presses, 2001.

Davidson, Cathy N. (ed.), Reading in America, Baltimore/London, The Johns Hopkins University Press, 1989.

Duval, Gilles, Littérature de colportage et imaginaire collectif en Angleterre à l'époque des Dicey (1720-v.1800), Talence, Presses universitaires de Bordeaux, 1991.

Fox, Adam, "Ballads, Libels, and Popular Ridicule in Jacobean England", Past and Present, 145 (1994), p. 47-83.

Graham, Judith, S., Puritan Family Life: The Diary of Samuel Sewall, Boston, Northeastern University Press, 2000.

Hall, David, D., Worlds of Wonder, Days of Judgment. Popular Religious Belief in Early New England, Cambridge (MA), Harvard University Press, 1990.

Hill, Christopher, A Turbulent, Seditious, and Factious People: John Bunyan and his Church 1628-1688, Oxford, Clarendon Press, 1988.

Houston, Robert A., Literacy in Early Modern Europe, London, Longman, 1988. 
Kaestle, Carl F., Pillars of the Republic: Common Schools and American Society, 1780-1860, New-York, Hill and Wang, 1983.

Laqueur, Thomas W., Religion and Respectability: Sunday Schools and Working Class Culture, 1780-1850, New Haven/London, Yale University Press, 1976.

Leavis, Queenie Dorothy, Fiction and the Reading Public, London, Pimlico, [1932], 2000.

Lemay, Leo J. A., Zall, Paul M. (eds), Benjamin Franklin's autobiography: an authoritative text, backgrounds, criticism, Toronto, Bantam Books, 1982.

Mayer, Robert, «Nathaniel Crouch, Bookseller and Historian: Popular History and Cultural Power in Late XVII ${ }^{\text {th }}$ Century England", XVIII $^{\text {th }}$ Century Studies, 27 (1994), p. 391-419.

Monaghan, Jennifer E., "Family Literacy in Early $18^{\text {th }}$ Century Boston, Cotton Mather and His Children", Reading Research Quarterly, 26 (1991/4), p. 342-370

-, Learning to Read and Write in Colonial America, Boston, University of Massachusetts Press, 2005.

Morgan, John, Godly Learning. Puritan Attitudes towards Reason, Learning and Education, Cambridge, Cambridge University Press, 1986.

Pedersen, Susan, "Hannah More Meets Simple Simon: Tracts, Chapbooks, and Popular Culture in Late Eighteenth Century England», The Journal of British Studies, 25 (1986), p. 84-113.

SPUfFORD, Margaret, "First Steps in Literacy: the Reading and Writing Experiences of the Humblest Seventeenth-Century Spiritual Autobiographers", in Literacy and Social Development in the West: a Reader, ed. by Harvey J. Graff, Cambridge, Cambridge University Press, 1981, p. 125-150.

-, Small Books and Pleasant Histories: Popular Fiction and its Readership in Seventeenth Century England, Cambridge, Cambridge University Press, 1981.

—, "Women Teaching Reading to Poor Children in the $16^{\text {th }}$ and $17^{\text {th }}$ centuries", in Opening the Nursery Door: Reading, writing and childhood 1600-1900, ed. by Mary Hilton, Morag Styles, Victor Watson, London/New York, Routledge, 1997.

Stannard, David E., The Puritan Way of Death, Oxford, Oxford University Press, 1977. 
Thompson, Edward Palmer, Customs in Common: Studies in Traditional Popular Culture, New York, The New Press, 1991.

Vincent, David, Bread, Knowledge and Freedom. A Study of Nineteenth Century Working Class Autobiography, London/New York, Methuen, 1981.

—, Literacy and Popular Culture: England 1750-1914, Cambridge, Cambridge University Press, 1993.

—, "Reading Made Strange. Context and Method in Becoming Literate in Eighteenth and Nineteenth Century in England", in Silences and Images. The Social History of the Classroom, ed. by Ian Grosvenor, Martin Lawn, Kate Rousmaniere, New York, Peter Lang, 1999, p. 181-197.

Watt, Tessa, Cheap print and popular piety, 1550-1640, Cambridge, Cambridge University Press, 1991.

\section{Crédits iconographiques}

Pages de garde de The Pilgrim's Progress de John Bunyan (1679):

Illustration récupérée sur le site de la British Library: http://www.bl.uk/learning/timeline/item126922.html 
